\title{
La gestión ambiental en los gobiernos locales en América Latina
}

\author{
Lozano Gómez, Percy \\ pelogo83@gmail.com \\ Escuela de Posgrado \\ Universidad César Vallejo \\ ORCID: 0000-0001-6951-4163 \\ Barbarán Mozo, Hipólito Percy \\ pbarmozito@hotmail.com \\ Escuela de Posgrado \\ Universidad César Vallejo \\ ORCID: 0000-0002-9316-202X
}

\section{RESUMEN}

La gestión ambiental comprende planes, políticas, herramientas, acciones y procesos que deben ser ejecutados de modo planificado, coordinado, sistemático y descentralizado con el propósito de potenciar la participación activa de los diversos actores locales para garantizar que las decisiones tomadas por las autoridades contribuyen con el cuidado y conservación del medioambiente, y así poder contribuir a su vez con el desarrollo sostenible de la localidad. El estudio tuvo un enfoque cualitativo, tipo básico y diseño teórico de revisión sistémica, estuvo constituido por 13 artículos científicos que representaron el fundamento bibliográfico del estudio, los mismos que fueron extraídos de la base de datos de Redalyc y Scielo, y para su búsqueda se utilizó como palabra clave gestión ambiental y gestión ambiental en gobiernos locales. Posteriormente a la revisión y análisis de los artículos se llegó a concluir que la mayoría de los gobiernos locales en América Latina se presentan diversos inconvenientes que afectan de manera directa y significativa el desarrollo de una buena gestión ambiental, pues la ausencia de planes y políticas, así como la falta de interés de las autoridades, etc., impide que se implementen mecanismos que promuevan el desarrollo íntegro de los pobladores dentro de un ambiente saludable.

Palabras clave: Gestión ambiental, gobierno local, América Latina. 


\title{
Environmental management in local governments in Latin America
}

\begin{abstract}
Environmental management comprises plans, policies, tools, actions and processes that must be executed in a planned, coordinated, systematic and decentralized manner in order to promote the active participation of the various local actors to guarantee that the decisions made by the authorities contribute to the care and conservation of the environment, and thus be able to contribute in turn to the sustainable development of the town. The study had a qualitative approach, basic type and theoretical design of a systemic review, it consisted of 13 scientific articles that represented the bibliographic basis of the study, which were extracted from the Redalyc and Scielo database, and were searched for used as a keyword environmental management and environmental management in local governments. After reviewing and analyzing the articles, it was concluded that the majority of local governments in Latin America have various problems that directly and significantly affect the development of good environmental management, since the absence of plans and policies, as well as the lack of interest of the authorities, etc., prevents the implementation of mechanisms that promote the integral development of the inhabitants within a healthy environment.
\end{abstract}

Keywords: Environmental management, local government, Latin America.

Artículo recibido: 25 dic. 2020 Aceptado para publicación: 26 ene. 2021

Correspondencia pelogo83@gmail.com Conflictos de Interés: Ninguna que declarar 


\section{INTRODUCCIÓN}

Durante los últimos años, el mundo ha venido sobrellevando constantes complicaciones ambientales generadas como consecuencia de los cambios que se producen con la creación de nuevas tecnologías, siendo el consumo excesivo y la falta de conciencia ambiental los factores más relevantes que originan el crecimiento acelerado de problemas ambientales y el deterioro progresivo de la calidad ambiental, reflejando de esta manera altos niveles de contaminación del planeta. Por lo tanto, es fundamental que las autoridades de los gobiernos municipales asuman con mayor responsabilidad y compromiso sus funciones correspondientes a la protección, conservación y manejo de los recursos naturales a través de la implementación de estrategias y/o alternativas que brinden solución y se encuentre direccionados a la prevención del deterioro acelerado de los mismos, para ello será también importante la participación responsable entre los municipios y las organizaciones ciudadanas para poder hacer frente al incremento de los problemas ambientales que se vienen suscitando en la actualidad (Toledo, 2017). En tanto Páez, et al. (2018), aluden que actualmente la gestión ambiental se ha categorizado como aquella estrategia de gran realce e importancia en el proceso organizacional y desempeño ambiental que tiene como principal propósito mejorar cada uno de las actividades ecoeficiente que contribuyan en la mitigación extrema de los impactos negativos originados por la contaminación o degradación del medio ambiente, cuyas principales causas se debido a la irresponsabilidad consciente de las empresas y ciudadanos al realizar malas prácticas en cuanto al uso irracional de los recursos naturales. Queda claro que velar por la protección y resguardo de los recursos naturales y el medio ambiente es uno de los retos de mayor importancia que tienen las autoridades municipales en conjunto con la ciudadanía para lograr la reestructuración de una óptima calidad ambiental y la mejora de los recursos naturales que permita de esa manera satisfacer las necesidades básicas (Gil-Rodríguez et al., 2020). Razón por la cual muchos países de todo el mundo vienen implementando nuevas actividades, mecanismos o acciones ecoeficientes que permita resguardar el medio ambiente y garantizar la administración y uso racional de los recursos naturales, tal es el caso de Cuba, que con el fin de salvaguardar el medio ambiente crearon bases para desarrollar el Sistema de Gestión Ambiental, cuyo principal objetivo y finalidad será coadyuvar en la recuperación óptima de los recursos que brinda la naturaleza a través de su conservación, mejoramiento y monitoreo del medio ambiente (Rodríguez \& Peña, 2019) 
En Perú las organizaciones e instituciones encargados y responsables de velar por el cuidado del medio ambiente son el Ministerio del Ambiente, el Organismo de Evaluación y Fiscalización Ambiental (OEFA), el Tribunal de Fiscalización Ambiental y las autoridades ambientales sectoriales, cuya principal función es fiscalizar y dictaminar el cumplimiento de las diversas normas ambientales establecidas por ley. Durante el periodo 2019, en el marco de la Política Nacional de Competitividad y Productividad cuyo propósito es generar bienestar para la población peruana por encima de la base de un crecimiento económico sostenible con enfoque territorial, se aprueban el Plan Nacional de Competitividad y Productividad, como también el Plan Nacional de Infraestructura para la Competitividad, que tienen como objetivo principal la incorporación de medidas asertivas que promuevan la sostenibilidad ambiental en la ejecución de actividades económicas, tales como estrategias de financiamiento frente al cambio climático, la gestión integral de residuos sólidos, la economía circular y acuerdos de producción limpia en los sectores que generan mayor contaminación (industrial, pesca y agricultura), estrategias de energía renovable, entre otros, quedando claro que dichos planes permitirán transitar de un desarrollo económico a un desarrollo sostenible o verde, sin embargo, de acuerdo a la realidad se conoce que la institucionalidad y la gestión ambiental del país ha estado restringida por las decisiones económicas para promover las inversiones que son necesarias, demostrando el poco interés por generar una calidad ambiental aceptable, vulnerando de esta manera el derecho de todo ciudadano de vivir en un ambiente saludables (Vidal, 2020). El análisis de la realidad presentada evoca una necesidad de abordar una revisión a nivel de estudios orientados a la gestión ambiental, en sus paradigmas, dimensiones o componentes, en ese sentido se orienta a una interrogante muy concreta ¿Cuáles son los principales hallazgos sobre la gestión ambiental en los gobiernos locales en América Latina?, de este modo alcanzar a evaluar la gestión ambiental en los gobiernos locales en América Latina.

\section{MATERIALES Y MÉTODOS}

En el estudio se consideró un enfoque cualitativo debido a que identificaron, analizaron e interpretaron diversos casos en los cuales se desarrolla la gestión ambiental con el objeto de facilitar la comprensión subjetiva sobre el tema (Sánchez, 2019). Asimismo, el artículo fue de tipo básico porque se estudiaron distintos enfoques conceptuales concernientes a la gestión ambiental dentro de un contexto latinoamericano (Ramírez, Meneses y Florez, 2013). De igual manera, presentó un diseño teórico de revisión sistémica pues la 
información recopilada fue correctamente analizada e interpretada en un periodo de tiempo concreto (Alto y Vallejo, 2015). Por otro lado, se ha tomado en cuenta que los participantes, como manifiesta Otzen y Manterola (2017) sostienen que los participantes son todos aquellos elementos que permiten adquirir información y datos relevantes para el desarrollo del estudio, cuyas cualidades son semejantes y por tanto facilitarán el análisis del objeto en estudio. En ese sentido, la muestra estuvo conformada por 13 artículos expuestos en la parte de los resultados, los mismos que están referidos a la gestión ambiental en los gobiernos locales. En tanto con la finalidad de facilitar la búsqueda de los artículos fue indispensable emplear la base de datos de Scielo y Redalyc, utilizando como palabras clave "Gestión ambiental", posteriormente se procedió a seleccionar aquellos artículos que se encuentran entre los años 2015 y 2020, escogiendo a los artículos que exclusivamente abarcaban la gestión ambiental. Para el estudio el instrumento empleado fue la matriz o tabla de registro elaborado en función a las principales características de los artículos, dentro de las cuales destaca el autor, año, tipo/diseño y conclusiones; donde tales criterios permitirán analizar la información presentada como resultados (Ñaupas, Valdivia, Palacios y Romero, 2018). Finalmente, para la elaboración del estudio se respetaron los lineamientos estipulados en las Normas APA $7^{\mathrm{a}}$ edición con el propósito de realizar las citaciones de manera correcta al momento de presentar información de distintos autores que hacen alusión sobre el tema en cuestión, respetando en todo momento los derechos de autor. De igual manera, se respetó las pautas dadas por la Universidad durante todo el proceso de elaboración y desarrollo del presente estudio.

\section{RESULTADOS Y DISCUSIÓN}

Mediante la revisión efectuada, se procede a presentar la siguiente tabla como parte de los resultados:

\begin{tabular}{|c|c|c|c|c|c|}
\hline $\mathbf{N}^{\circ}$ & Autor & País & Clasificación & Tipo y diseño & Conclusiones \\
\hline 1 & $\begin{array}{l}\text { Carrillo y } \\
\text { Casellas } \\
(2016)\end{array}$ & Colombia & $\begin{array}{l}\text { Artículo } \\
\text { científico }\end{array}$ & $\begin{array}{l}\text { No } \\
\text { experimental, } \\
\text { Descriptivo }\end{array}$ & $\begin{array}{l}\text { La gestión ambiental presenta una serie de } \\
\text { deficiencias, por cuanto no se consideran } \\
\text { los componentes internos del país al } \\
\text { momento de diseñar los planes y políticas } \\
\text { ambientales. Así pues, entre las principales } \\
\text { falencias que afectan la gestión en los } \\
\text { gobiernos locales destaca: i) Falta de } \\
\text { capacidades económicas y humanas, } \\
\text { porque las municipalidades no disponen de } \\
\text { fondos necesarios para implementar } \\
\text { mecanismos que contribuyan con una }\end{array}$ \\
\hline
\end{tabular}




\begin{tabular}{|c|c|c|c|c|c|}
\hline & & & & & $\begin{array}{l}\text { gestión ambiental efectiva, además de que } \\
\text { los colaboradores no cuentan con las } \\
\text { aptitudes necesarias para llevar a cabo sus } \\
\text { actividades de modo pertinente; ii) Falta de } \\
\text { voluntad política, el cual refleja la ausencia } \\
\text { de interés y empeño por parte de las } \\
\text { autoridades locales y colaboradores en el } \\
\text { seguimiento de acciones que contribuyan } \\
\text { con la buena gestión ambiental; iii) Captura } \\
\text { de órganos de decisión por parte de la élite } \\
\text { local, debido a que el Estado no otorga el } \\
\text { poder y autonomía necesaria para que los } \\
\text { municipios puedan tomar buenas } \\
\text { decisiones de manera independiente en } \\
\text { bienestar de la población local. }\end{array}$ \\
\hline 2 & $\begin{array}{l}\text { Hernánd } \\
\text { ez (2015) }\end{array}$ & Venezuela & $\begin{array}{l}\text { Artículo } \\
\text { científico }\end{array}$ & $\begin{array}{l}\text { No } \\
\text { experimental, } \\
\text { Descriptivo }\end{array}$ & $\begin{array}{l}\text { Se reconoce la importancia de llevar a cabo } \\
\text { una gestión ambiental eficiente con el } \\
\text { propósito de conocer el manejo de los } \\
\text { recursos naturales y humanos por parte de } \\
\text { los gobiernos locales, el cual debe estar } \\
\text { orientado al desarrollo sustentable. } \\
\text { Además, se identificaron diversos } \\
\text { inconvenientes relacionados con la } \\
\text { aplicación de políticas públicas que } \\
\text { permitan dirigir, administrar y planear } \\
\text { acciones frente al impacto ambiental que } \\
\text { afecta a la localidad, por tal motivo se pudo } \\
\text { reconocer que existe un bajo nivel de } \\
\text { conciencia ambiental y desarrollo } \\
\text { sustentable por parte de los pobladores } \\
\text { locales. Finalmente, resulta relevante } \\
\text { señalar que, si bien es cierto, los municipios } \\
\text { de Venezuela buscan diseñar de manera } \\
\text { constante planes para el cuidado y mejora } \\
\text { del medioambiente, las acciones se llevan a } \\
\text { cabo de manera pausada, por lo que resulta } \\
\text { dificultoso que se presenten cambios } \\
\text { positivos de manera rápida dentro de la } \\
\text { localidad. }\end{array}$ \\
\hline 3 & $\begin{array}{l}\text { Escobar } \\
\text { y Romo } \\
(2019)\end{array}$ & México & $\begin{array}{l}\text { Artículo } \\
\text { científico }\end{array}$ & $\begin{array}{l}\text { No } \\
\text { experimental, } \\
\text { Transversal }\end{array}$ & $\begin{array}{l}\text { Como bien se sabe que la gestión ambiental } \\
\text { dentro de un contexto local busca seguir } \\
\text { una serie de procesos para que se acepten y } \\
\text { tomen decisiones de manera consciente } \\
\text { ante una situación ambiental poco } \\
\text { favorable caracterizado por el incremento } \\
\text { de la industrialización, desarrollo } \\
\text { económico acelerado y problemas } \\
\text { ambientales; frente a ello es necesario que }\end{array}$ \\
\hline
\end{tabular}




\begin{tabular}{|c|c|c|c|c|c|}
\hline & & & & & $\begin{array}{l}\text { los municipios desarrollen una gestión } \\
\text { ambiental proactiva de tal manera que se } \\
\text { puedan implementar medidas o } \\
\text { mecanismos que permitan anticiparse a las } \\
\text { deficiencias concernientes al aspecto } \\
\text { ambiental del territorio. En efecto, se puede } \\
\text { deducir que para garantizar una gestión } \\
\text { ambiental efectiva es fundamental evaluar } \\
\text { el origen multicausal de los problemas que } \\
\text { se presentan dentro de un contexto } \\
\text { ambiental y posteriormente diseñar } \\
\text { medidas correctivas a fin de que, por medio } \\
\text { de la participación activa de los actores, se } \\
\text { pueda dar solución a tales inconvenientes. }\end{array}$ \\
\hline 4 & $\begin{array}{l}\text { Cerati y } \\
\text { Queiroz } \\
(2016)\end{array}$ & Brasil & $\begin{array}{l}\text { Artículo } \\
\text { científico }\end{array}$ & Descriptivo & $\begin{array}{l}\text { Se reconoce que la participación social } \\
\text { representa un componente esencial para } \\
\text { llevar a cabo una gestión ambiental } \\
\text { efectiva, por cuanto es necesaria la } \\
\text { intervención de los individuos u } \\
\text { organismos contribuyen con la rápida y } \\
\text { oportuna identificación y reconocimiento } \\
\text { de los problemas o posibles problemas que } \\
\text { podrían representar un suceso negativo } \\
\text { mayor y afectar el desarrollo sostenible de } \\
\text { la localidad. Es así que, dentro de los } \\
\text { principales efectos suscitados por los } \\
\text { problemas ambientales destaca la } \\
\text { degradación de las áreas verdes, puesto que } \\
\text { la expansión poco ordenada y la falta de } \\
\text { planificación y organización de la } \\
\text { infraestructura de las zonas urbanas por } \\
\text { parte de los funcionarios y colaboradores } \\
\text { del gobierno local ha generado que las } \\
\text { áreas verdes de la localidad se encuentren } \\
\text { en un estado de deterioro. }\end{array}$ \\
\hline 5 & $\begin{array}{l}\text { Castro y } \\
\text { Rajadel } \\
(2015)\end{array}$ & Cuba & $\begin{array}{l}\text { Artículo } \\
\text { científico }\end{array}$ & Descriptivo & $\begin{array}{l}\text { El municipio de Cumanayagua presenta un } \\
\text { alto nivel de complejidad para llevar a cabo } \\
\text { una gestión ambiental adecuada debido a } \\
\text { que los funcionarios y colaboradores } \\
\text { reconocen una serie de deficiencias dentro } \\
\text { de las cuales se encuentra la falta de } \\
\text { implementación de acciones que } \\
\text { contribuyan con la programación y } \\
\text { ejecución efectiva de proyectos, además de } \\
\text { reconocerse que en ciertas ocasiones los } \\
\text { proyectos no están orientados a solucionar } \\
\text { problemas ambientales prioritarios que } \\
\text { afectan el desarrollo sostenible de la }\end{array}$ \\
\hline
\end{tabular}




\begin{tabular}{|c|c|c|c|c|c|}
\hline & & & & & $\begin{array}{l}\text { localidad. Asimismo, se reconoce que los } \\
\text { colaboradores no cuentan con una serie de } \\
\text { procesos y mecanismos que contribuyan } \\
\text { con la eficiente gestión ambiental local. En } \\
\text { ese sentido, se reconoce que tanto el } \\
\text { municipio de Cumanayagua y Abreus } \\
\text { perciben deficiencias durante el desarrollo } \\
\text { de sus actividades de gestión ambiental } \\
\text { debido a que las medidas correctivas no } \\
\text { están siendo aplicadas de manera efectiva } \\
\text { puesto que no solucionan los problemas } \\
\text { ambientales presentados en el ámbito local. }\end{array}$ \\
\hline 6 & $\begin{array}{l}\text { Vásquez } \\
\text { y } \\
\text { Pañuela } \\
(2015)\end{array}$ & Colombia & $\begin{array}{l}\text { Artículo } \\
\text { científico }\end{array}$ & Descriptivo & $\begin{array}{l}\text { La gestión ambiental en los gobiernos } \\
\text { locales colombianos comprende una serie } \\
\text { de componentes, siendo uno de los } \\
\text { fundamentales el adecuado control social, } \\
\text { correcto ordenamiento territorial, adecuada } \\
\text { planeación, toma de decisiones asertiva, } \\
\text { etc., puesto que estos permiten establecer } \\
\text { un marco institucional como parte de la } \\
\text { gestión ambiental, la misma que facilitará } \\
\text { el desarrollo local sostenible. Cabe precisar } \\
\text { que la creación de un espacio que } \\
\text { promueva la interacción entre los } \\
\text { pobladores y el municipio facilitará el } \\
\text { abordaje adecuado del problema ambiental } \\
\text { que se presenta en la localidad, el mismo } \\
\text { que está afectando de manera directa y } \\
\text { significativa la convivencia de los } \\
\text { pobladores. Sin embargo, para ello resulta } \\
\text { necesario hacer frente a los diversos } \\
\text { cambios relacionados a la intervención } \\
\text { pública y control administrativo en los } \\
\text { gobiernos locales. }\end{array}$ \\
\hline 7 & $\begin{array}{l}\text { Toledo } \\
\text { (2017) }\end{array}$ & El salvador & $\begin{array}{l}\text { Artículo } \\
\text { científico }\end{array}$ & Descriptivo & $\begin{array}{l}\text { La gestión ambiental representa a todos los } \\
\text { planes organizados según las metas } \\
\text { propuestas, ello de acuerdo a los proyectos } \\
\text { que se priorizan en una secuencia temporal } \\
\text { teniendo en cuenta los indicadores de } \\
\text { logros acordes a los periodos de tiempo } \\
\text { señalado. Asimismo, se pudo reconocer } \\
\text { que el nivel de capacitación es bajo, pues el } \\
33 \% \text { de los encuestado no tienen estudios } \\
\text { superiores; por otro lado, el } 83 \% \text { menciona } \\
\text { que el nivel de infraestructura de la } \\
\text { institución es alto; además, el } 50 \% \text { opina } \\
\text { que el nivel de calidad de los materiales y } \\
\text { equipos es alto; también, el } 87 \% \text { afirman }\end{array}$ \\
\hline
\end{tabular}




\begin{tabular}{|c|c|c|c|c|c|}
\hline & & & & & $\begin{array}{l}\text { que el nivel de efectividad de las normas } \\
\text { ambientales que regulan las actividades } \\
\text { específicas es alto debido a que gran parte } \\
\text { de estas abarcan un contexto general; de } \\
\text { igual manera, el } 83 \% \text { cuenta con un plan de } \\
\text { gestión ambiental pero la falta de } \\
\text { conocimiento suscita que no puedan ser } \\
\text { ejecutados correctamente; por último, se } \\
\text { evidencia que el } 50 \% \text { de los gobiernos } \\
\text { locales cuentan con instrumentos de } \\
\text { políticas ambientales. }\end{array}$ \\
\hline 8 & $\begin{array}{l}\text { Figueroa } \\
\text { y Castillo } \\
(2017)\end{array}$ & México & $\begin{array}{l}\text { Artículo } \\
\text { científico }\end{array}$ & Descriptivo & $\begin{array}{l}\text { Los municipios presentan diversas } \\
\text { falencias durante el seguimiento de las } \\
\text { actividades y proyectos medioambientales, } \\
\text { siendo uno de los más significativos la falta } \\
\text { de control y monitoreo de la legislación } \\
\text { ambiental, por cuanto ello dificulta que se } \\
\text { pueda constatar la correcta actuación de los } \\
\text { actores ambientales. Frente a tales } \\
\text { problemas que ponen en riesgo la } \\
\text { estabilidad y equilibrio medioambiental del } \\
\text { país, los municipios mexicanos } \\
\text { reconocieron la necesidad de elaborar } \\
\text { como propuesta una reforma dentro de un } \\
\text { marco jurídico de tal manera que se pueda } \\
\text { regular acciones coherentes e integrales por } \\
\text { parte de los servidores y funcionarios } \\
\text { públicos competentes con el propósito de } \\
\text { plantear modificaciones en las funciones } \\
\text { que tienen los mismos y así expandir el } \\
\text { poder económico y político para garantizar } \\
\text { el seguimiento de actividades que } \\
\text { prevengan y controlen el nivel de } \\
\text { contaminación ambiental que existe en las } \\
\text { localidades. }\end{array}$ \\
\hline 9 & $\begin{array}{l}\text { Barcia } \\
(2018)\end{array}$ & Ecuador & $\begin{array}{l}\text { Artículo } \\
\text { científico }\end{array}$ & Descriptivo & $\begin{array}{l}\text { La falta de una buena gestión ambiental por } \\
\text { parte de las autoridades locales ocasiona } \\
\text { que se presenten una serie de problemas } \\
\text { que dificultan el cuidado y conservación } \\
\text { del medioambiente y además afecta de } \\
\text { manera significativa la integridad y } \\
\text { bienestar de los pobladores. Teniendo en } \\
\text { consideración que el medioambiente es de } \\
\text { interés público, ha quedado evidenciado la } \\
\text { omisión por parte de las autoridades locales } \\
\text { sobre el tema en cuestión, por tal motivo se } \\
\text { reconoce la importancia de que los } \\
\text { gobiernos locales garanticen una actuación }\end{array}$ \\
\hline
\end{tabular}




\begin{tabular}{|c|c|c|c|c|c|}
\hline & & & & & $\begin{array}{l}\text { efectiva respecto al cuidado del ambiente } \\
\text { con el propósito de asegurar la interacción } \\
\text { continua entre la sociedad civil y las } \\
\text { autoridades locales de tal manera que se } \\
\text { pueda velar en todo momento por la } \\
\text { protección de los derechos de la población } \\
\text { en el cual se establece que "Todas las } \\
\text { personas deben vivir dentro de un medio } \\
\text { ambiente sano sin ningún tipo de } \\
\text { contaminación". }\end{array}$ \\
\hline 10 & $\begin{array}{l}\text { Condori } \\
\text { y Reyna } \\
(2019)\end{array}$ & Perú & $\begin{array}{l}\text { Artículo } \\
\text { científico }\end{array}$ & $\begin{array}{l}\text { No } \\
\text { experimental, } \\
\text { Descriptivo- } \\
\text { Transversal }\end{array}$ & $\begin{array}{l}\text { La gestión ambiental en la institución } \\
\text { presenta un nivel deficiente en un } 68 \% \\
\text { debido a los diversos problemas que suelen } \\
\text { presentarse, uno de estos es la presencia de } \\
\text { vacíos legales dentro de las leyes y } \\
\text { normativas ambientales, por cuanto ello } \\
\text { dificulta que se pueda realizar un } \\
\text { seguimiento efectivo de las actividades } \\
\text { orientadas a garantizar un equilibrio } \\
\text { medioambiental; además, se reconoce que } \\
\text { las autoridades no están aplicando } \\
\text { pertinentemente las políticas y programas } \\
\text { que permitan difundir e implementar de } \\
\text { manera correcta las acciones ambientales } \\
\text { debido a la falta de conocimientos y } \\
\text { habilidades por parte de los funcionarios y } \\
\text { servidores competentes. Por tal motivo se } \\
\text { puede deducir que tales problemas afectan } \\
\text { de modo directo la conservación del } \\
\text { medioambiente. }\end{array}$ \\
\hline 11 & $\begin{array}{l}\text { Campos } \\
\text { (2019) }\end{array}$ & Perú & $\begin{array}{l}\text { Artículo } \\
\text { científico }\end{array}$ & $\begin{array}{l}\text { Aplicada } \\
\text { Descriptivo- } \\
\text { Transversal- } \\
\text { Prospectivo }\end{array}$ & $\begin{array}{l}\text { La aparición de numerosas falencias en las } \\
\text { municipalidades de Huacrachuco ha } \\
\text { generado una serie de problemas respecto } \\
\text { al manejo de residuos sólidos, dentro de las } \\
\text { principales falencias se pueden precisar las } \\
\text { siguientes: Ausencia de instrumentos de } \\
\text { gestión ambiental concernientes a la } \\
\text { infraestructura de residuos sólidos; los } \\
\text { procesos para la obtención de autorización } \\
\text { se efectúan de manera inadecuada; } \\
\text { ausencia de compromiso por parte de las } \\
\text { autoridades de las municipalidades para } \\
\text { implementar plantas de tratamiento y crear } \\
\text { programas que ayuden a segregar, reutilizar } \\
\text { y reciclar residuos sólidos; la falta de } \\
\text { oportunidad en el pago por los servicios } \\
\text { públicos dentro de los cuales destaca la } \\
\text { limpieza pública; las municipales no }\end{array}$ \\
\hline
\end{tabular}




\begin{tabular}{|c|c|c|c|c|c|}
\hline & & & & & $\begin{array}{l}\text { utilizan herramientas, mecanismos e } \\
\text { instrumentos que coadyuven con la } \\
\text { recolección de los residuos sólidos; } \\
\text { ausencia de una ruta establecida para el } \\
\text { traslado de residuos tóxicos; por lo tanto, se } \\
\text { puede reconocer que todo ello dificulta una } \\
\text { correcta actuación de las autoridades } \\
\text { municipales. }\end{array}$ \\
\hline 12 & $\begin{array}{l}\text { Tumi y } \\
\text { Escobar } \\
(2018)\end{array}$ & Perú & $\begin{array}{l}\text { Artículo } \\
\text { científico }\end{array}$ & $\begin{array}{l}\text { No } \\
\text { experimental- } \\
\text { Transversal- } \\
\text { Documental- } \\
\text { Descriptivo }\end{array}$ & $\begin{array}{l}\text { Las municipalidades de Puno reconocen la } \\
\text { aparición de diversas falencias, dentro de } \\
\text { las cuales se encuentran las siguientes: } \\
\text { Deficiente gestión de las aguas residuales, } \\
\text { puesto que sólo el } 17 \% \text { cumple con las } \\
\text { regulaciones establecidas; Deficiente } \\
\text { gestión de residuos sólidos, debido a que no } \\
\text { cuentan con programas y planes que } \\
\text { contribuyan con el correcto manejo de tales } \\
\text { residuos; Ausencia de control respecto al } \\
\text { incremento del nivel de contaminación del } \\
\text { ambiente con metales pesados y } \\
\text { plaguicidas, principalmente de metales } \\
\text { como cobre, plomo, cadmio, etc.; } \\
\text { deficiente control en el incremento de las } \\
\text { actividades mineras e incremento del nivel } \\
\text { de contaminación del medioambiente, } \\
\text { porque la minería informal suscita un } \\
\text { movimiento de las tierras; ausencia de } \\
\text { medidas frente al cambio climático, pues } \\
\text { no se toman medidas frente al incremento } \\
\text { de las temperaturas y los cambios que ello } \\
\text { podría ocasionar tales como las } \\
\text { inundaciones, sequías, heladas, entre otros; } \\
\text { ausencia de planes ante el deterioro de la } \\
\text { biodiversidad, lo cual ocasiona un aumento } \\
\text { del deceso de especies nativas en peligro de } \\
\text { extinción. }\end{array}$ \\
\hline 13 & $\begin{array}{l}\text { Abanto y } \\
\text { Cano } \\
(2015)\end{array}$ & Perú & $\begin{array}{l}\text { Artículo } \\
\text { científico }\end{array}$ & $\begin{array}{l}\text { Descriptivo- } \\
\text { Analítico }\end{array}$ & $\begin{array}{l}\text { Se reconoce la importancia de las } \\
\text { municipalidades en el desarrollo de una } \\
\text { buena gestión ambiental puesto que estas } \\
\text { asumen el rol de gestionar, fiscalizar, } \\
\text { promover y apoyar con la realización de } \\
\text { acciones en mejora del cuidado } \\
\text { medioambiental, todo ello con el propósito } \\
\text { de orientar el diseño e implementación de } \\
\text { un modelo de gestión ambiental integrada } \\
\text { con el objeto de garantizar la sostenibilidad } \\
\text { del medioambiente. Además, se percibe } \\
\text { que la ausencia de procesos sistemáticos y }\end{array}$ \\
\hline
\end{tabular}




\begin{tabular}{|l|l|l|l|}
\hline & & $\begin{array}{l}\text { toma de decisiones poco asertivas dificulta } \\
\text { el control y seguimiento de las acciones } \\
\text { ambientales en pro del cuidado y } \\
\text { conservación del ambiente. Frente a tales } \\
\text { inconvenientes las municipalidades } \\
\text { reconocen la importancia de abarcar los } \\
\text { distintos niveles de gobierno con la } \\
\text { finalidad de asegurar la consecución de las } \\
\text { metas y promoción de una adecuada } \\
\text { gestión ambiental. }\end{array}$ \\
\hline
\end{tabular}

Entre los resultados presentados en la tabla anterior prevalecen aquellos artículos desarrollados en el Perú puesto que se seleccionaron 4 artículos, y seguidamente 2 artículos fueron provenientes de Colombia y 2 artículos de México; cabe precisar además que estos hacen referencia a la forma en la que se desarrolla la gestión ambiental dentro de los gobiernos locales, y los diseños de investigación fueron principalmente descriptivo debido a que 12 de estos se acogieron a ese diseño, mientras que 5 presentaron un diseño no experimental y 4 fueron transversales.

En efecto, a través de la revisión y análisis de los artículos científicos relacionados a la gestión ambiental se pudo percibir que los municipios y municipalidades reconocen la necesidad de diseñar e implantar herramientas y planes que coadyuven con la efectividad de la gestión ambiental debido a que ello permitirá que tales instituciones puedan llevar a cabo un correcto seguimiento, control y evaluación de las políticas ambientales para que los pobladores no sean afectados en gran medida, garantizando a su vez un crecimiento y desarrollo local sostenible. Es así que, dentro de un contexto internacional, se pudo identificar que los principales inconvenientes que presentan los municipios durante el desarrollo de la gestión ambiental son: La falta de efectividad de planes y políticas ambientales porque, si bien es cierto que gran parte de los municipios cuentan con políticas y planes reguladores de las actividades y proyectos que deben ejecutarse en un contexto local, la carencia de aptitudes de las autoridades y servidores dificulta que estos puedan realizar un seguimiento efectivo de los mismos; La ausencia de implementación de medidas estratégicas que contribuyan con el cuidado o reduzcan el daño hacia el medioambiente, puesto que ello dificulta que se pueda tomar conciencia sobre el impacto negativo que ocasiona la ejecución de una determinada actividad o proyecto; bajo nivel de promoción de la participación activa de los pobladores, pues la falta de conocimiento respecto a la percepción de los pobladores sobre los problemas ambientales dificulta que apliquen 
medidas pertinentes frente a los mismos. Por otro lado, en Perú, las principales deficiencias concernientes a la gestión ambiental que se presentan dentro de las municipalidades son: Ausencia de normativas y legislaciones claras y precisas, pues ello ocasiona que las autoridades municipales presenten dificultades durante el seguimiento pertinente de las acciones ambientales; Falta de implementación y uso de herramientas que contribuyan con el control y manejo de los residuos sólidos, aguas residuales, metales pesados y plaguicidas, así como la falta de monitoreo de actividades ilícitas, ello suscita que no se reduzcan los niveles de contaminación existentes.

El presente artículo planteó como objetivo general evaluar la gestión ambiental en los gobiernos locales en América Latina, por tal motivo se procede a exponer los hallazgos más relevantes conforme a la revisión sistémica realizada.

Inicialmente, respecto a las características principales de los artículos seleccionados como participantes, se pudo conocer que predominaron los artículos peruanos con 4, siendo seguidos por Colombia y México pues se consideraron como objeto de análisis 2 artículos correspondientes a cada uno de los países. Bajo esa premisa resulta pertinente destacar la afirmación de Miguel (2011), pues el autor deduce que la elaboración y distribución de artículos científicos en América Latina no es uniforme debido a una serie de factores que inciden en la realidad que estudian cada uno de los investigadores. Por otra parte, en cuanto al tipo y diseño de investigación, se reconocen que la mayoría de los artículos emplearon diseños no experimentales, a nivel descriptivo, documental y transversales.

Estos resultados son congruentes con lo sostenido por Ramírez y Arias (2018), puesto que los autores sostienen que la producción de artículos está orientado principalmente al diseño no experimental porque los investigadores no buscan implementar mecanismos o herramientas que solucionen la problemática que estudian, sino que se centran principalmente en recopilar información sobre la problemática de tal manera que se identifiquen y analicen las principales causas y efectos que originan la misma.

Asimismo, se pudo conocer la presencia de diversas deficiencias e inconvenientes vinculados a la gestión ambiental que se presentan dentro de los gobiernos locales en América Latina, dentro de las cuales destaca la ausencia de planes y políticas ambientales efectivas, falta de conocimientos y preparación de las autoridades y servidores públicos locales, falta de acciones y medidas estratégicas que permitan asegurar el cuidado y 
reducción de daño medioambiental, ausencia de participación activa de los pobladores, entre otras deficiencias.

Los resultados presentaron limitaciones debido a que sólo se consideró el idioma español, porque los países de América Latina emplearon tal idioma durante la realización de la investigación; otra de las limitaciones fue la escasez de la base de datos respecto al tema en investigación; del mismo modo, para la depuración de la información se tuvo que aplicar ciertos de inclusión y exclusión de tal manera que se seleccione y se efectúe la revisión correspondiente de una cantidad prudente de artículos científicos. Estos resultados son congruentes a los expuestos por Carrillo y Casellas (2016), por cuanto los autores aseveran que los estudios realizados en Bolivia, Brasil, Guatemala, México y Nicaragua permitieron conocer que los gobiernos locales de países latinoamericanos atraviesan por diversos inconvenientes al momento de llevar a cabo las actividades correspondientes a la gestión ambiental.

Por último, después de haber analizado y constatado la información, el presente artículo estuvo enfocado a adquirir mayores conocimientos relacionados a la gestión ambiental en los gobiernos locales en América Latina, puesto que la coyuntura actual exige el reconocimiento de un estudio que se oriente en la mayor medida de lo posible en la realidad actual, la misma que se encuentra en constante cambio como producto de las variaciones que se presentan dentro del medioambiente.

\section{CONCLUSIÓN}

Como parte de los principales hallazgos sobre la gestión ambiental en los gobiernos locales en América Latina, se evidencia la presencia de diversas deficiencias e inconvenientes vinculados a su gestión, dentro de las cuales destaca la ausencia de planes y políticas ambientales efectivas, falta de conocimientos y preparación de las autoridades, servidores públicos locales, falta de acciones y medidas estratégicas que permitan asegurar el cuidado y reducción de daño medioambiental.

Frente a esta problemática, se reconoce la necesidad de diseñar e implementar mecanismos y herramientas que contribuyan con el incremento del potencial y desempeño de los colaboradores y servidores, mejoren el control de instrumentos, recursos materiales y económicos con la finalidad de garantizar su uso eficiente de los mismos para mejorar la solución de los problemas ambientales, así como la consecución de los objetivos y programas ambientales. 


\section{LISTA DE REFERENCIAS}

Abanto, G. y Cano, E. (2015). Gestión ambiental integrada y desarrollo sostenible para el Plan de Desarrollo Regional Concertado de La Libertad 2010-2021. Revista Ciencia y Tecnología. $11(3)$

$9-15$. https://revistas.unitru.edu.pe/index.php/PGM/article/view/1111

Alto, M. \& Vallejo, G. (2015). Diseño de investigación en psicología. España: Ediciones Pirámide

Barcia, S. (2018). Gestión ambiental de la Fiscalía Provincial en Ecuador: Una mirada desde la Constitución Ecuatoriana. Telos. 20(2), 1-17. https://www.redalyc.org/jatsRepo/993/99356889006/99356889006.pdf

Campos, C. (2019). Gestión de residuos sólidos municipales en el distrito de HuacrachucoMarañón: Una propuesta de mejora. Gaceta Científica. 5(2), 85-92. http://revistas.unheval.edu.pe/index.php/gacien/article/view/690

Carrillo, P. y Casellas, A. (2016). Descentralización y gestión ambiental en América Latina: Un análisis de las publicaciones académicas. Cuadernos de Desarrollo Rural. 13(78), 67-96. https://www.redalyc.org/pdf/117/11753256003.pdf

Castro, N. y Rajadel, O. (2015). El desarrollo local, la gestión de gobierno y los sistemas de innovación. Revista Universidad y Sociedad. 7(1), 63-72. http://scielo.sld.cu/scielo.php?script=sci_arttext\&pid=S2218-36202015000100009

Cerati, T. y Queiroz, A. (2016). Participación social en la gestión ambiental: Estudio de caso en una unidad de conservación urbana en el municipio de Sao Paulo, Brasil. Estudios demográficos y urbanos. Estudios demográficos urbanos. 31(1), 87-113. http://www.scielo.org.mx/scielo.php?script=sci_arttext\&pid=S018672102016000100087

Condori, M. y Reyna, G. (2019). Percepción de la responsabilidad social universitaria en estudiantes de la Facultad de Sociología de una universidad pública de la ciudad de Huancayo, Perú. Revista $\quad$ Espacios. https://www.revistaespacios.com/a19v40n39/a19v40n39p08.pdf

Escobar, R. y Romo, M. (2019). Los instrumentos de planeación local y la prevención de desastres: Un análisis de gestión ambiental transversal en Mexicali, Baja California. Revista Iberoamericana de Estudios Municipales. 1(20), 113-147. http://dx.doi.org/10.4067/S0719-17902019000200113 
Figueroa, L. y Castillo, R. (2017). Gestión ambiental. Revista Alegatos. 1(31), 1-8. http://alegatos.azc.uam.mx/index.php/ra/article/view/1263

Gil-Rodríguez, A., Pell-del Río, S. y Valdés-Santiago, D. (2020). Guía metodológica para la gestión ambiental: Una propuesta cubana. Revista Cubana de Educación Superior. 39(2). http://scielo.sld.cu/pdf/rces/v39n2/0257-4314-rces-39-02-e13.pdf

Hernández, E. (2015). Ambiente, gestión ambiental: Avances y retrocesos del ambiente y desarrollo sustentable en Venezuela. Revista Provincia. 1(34), 97-116. https://www.redalyc.org/pdf/555/55544729006.pdf

Miguel, S. (2011). Revistas y producción científica de América Latina y el Caribe: su visibilidad en Scielo, Redalyc y Scopus. Revista Interamericana de Bibliotecología. 34(2), 187-199. http://www.scielo.org.co/pdf/rib/v34n2/v34n2a6.pdf

Naupas, H., Valdivia, M., Palacios, J. y Romero, H. (2018). Metodología de la investigación cuantitativa-cualitativa y redacción de la tesis ( $5^{\mathrm{a}}$ ed.) Colombia: Ediciones de la U. https://n9.cl/0rgb3

Otzen, T. y Manterola, C. (2017). Técnica de muestreo sobre una población a estudio. International Journal of Morphology. 35(1), 227-232. http://dx.doi.org/10.4067/S0717-95022017000100037

Páez, J., Recalde, M, Zumarraga, K. y Haro, E. (2018). Nociones básicas de gestión ambiental. Ecuador: Editorial El Conejo. https://n9.cl/e67am

Ramírez, R., Meneses, J. \& Florez, M. (2013). Una propuesta metodológica para la conducción de revisiones sistemáticas de la literatura en la investigación biomédica. $\begin{array}{lllll}\text { CES Movimiento } & y & \text { 6alud. }\end{array}$ http://132.248.161.133:8080/jspui/handle/123456789/5467

Ramírez, T. y Arias, F. (2018). Doctores en educación y producción de artículos en revistas indexadas: El caso venezolano. Revista de Pedagogía. 39(105), 261-286. https://n9.cl/v0155

Rodríguez, A. \& Peña, L. (2019). La protección del medioambiente en Cuba, una prioridad gubernamental. Revista Novedades en Población. 15(39), 113-122. http://scielo.sld.cu/pdf/rnp/v15n30/1817-4078-rnp-15-30-113.pdf

Sánchez, F. (2019). Fundamentos epistemológicos de la investigación cualitativa y cuantitativa: Consensos y disensos. Revista Digital de Investigación en docencia universitaria. 13(1), 102-122. http://dx.doi.org/10.19083/ridu.2019.644 
Toledo, B. (2017). La importancia de la gestión ambiental municipal. Estudio de caso: Municipios del departamento de Santa Ana, El Salvador. INVENTUM, 12(23), 22-34. https://doi.org/10.26620/uniminuto.inventum.12.23.2017.22-34

Tumi, J. y Escobar, F. (2018). Incidencia de factores sociales y políticos en la inversión ambiental del Gobierno Regional de Puno-Perú. Revista de Investigaciones Altoandinas, 20(2), 235-250. http://dx.doi.org/10.18271/ria.2018.367

Vásquez, D. y Pañuela, M. (2015). La gobernabilidad local en el contexto de la nueva gestión pública: requisito para la gestión ambiental urbana. Revista Luna Azul, 1(39), 63-88. https://revistasojs.ucaldas.edu.co/index.php/lunazul/article/view/1760

Vidal, I. (2020). La protección ambiental: Si antes era una necesidad, hoy es una prioridad. Perú: LP Derecho. https://lpderecho.pe/la-proteccion-ambiental-si-antes-era-unanecesidad-hoy-es-una-prioridad/ 